\title{
HOW DO SATURN'S RADIO EMISSIONS RESPOND TO MAGNETOSPHERIC COMPRESSIONS AND TAIL RECONNECTION: AN ANALYSIS OF SKR BURST AND LOW FREQUENCY EXTENSIONS (LFES)
}

\author{
C. Jackman*, J. J. Reed*, D. Whiter*, L. Lamy ${ }^{\dagger}$, and W.S. Kurth ${ }^{\ddagger}$
}

\begin{abstract}
Saturn Kilometric Radiation has been shown to respond both to solar wind compressions and magnetotail reconnection events through bursts of the main emission [e.g. Taubenschuss et al., 2006, Ann. Geophys. 24, 3139-3150; Rucker et al., 2008, Adv. Space Res. 42, 40-47; Badman et al., 2008, Ann. Geophys. 26, 3641-3651] and extensions of the emission to lower frequencies (LFEs) [e.g. Jackman et al., 2009, JGR 114, A08211]. The LFEs in particular have been suggested to be associated with a growth/motion of the SKR source region to higher altitudes, analogous to a feature noted in the terrestrial AKR literature [e.g. Morioka et al., 2007, JGR 112, A06245; Morioka et al., 2008, JGR 113, A09213].

This presentation will review past work linking LFEs to magnetospheric dynamics and show results from a new study [Reed et al., 2016, in preparation] which focuses on Cassini RPWS and MAG data from the tail orbits of 2006. This comprehensive new survey aims to advance our ability to use LFEs as a remote proxy for dynamic magnetospheric events. An automated method to search for LFEs based on the statistical characteristics of the radio spectrum has been developed. Properties of LFEs such as their length and recurrence rate will also be discussed.

Finally the link between planetary periodicities (PPOs) at Saturn (as tracked by the SKR and MAG phases) and SKR bursts/LFEs will be shown, building on results from Jackman et al. [2016, JGR 121, 2922-2934] who examined the correlation between dynamic events in Saturn's tail and PPO phases.
\end{abstract}

\footnotetext{
* Department of Physics and Astronomy, University of Southampton, Southampton, UK

${ }^{\dagger}$ LESIA, Observatoire de Paris, Meudon, France

$\ddagger$ Department of Physics and Astronomy, University of Iowa, Iowa City, IA, USA
} 
